Revue des patrimoines

40 | 2019

Lits historiques. Première anthologie des lits européens du XVe au XIXe siècle

\title{
Les témoignages du lit de la chambre de la duchesse de Bourbon au Palais-Bourbon et leur présentation au Louvre
}

The Bed from the Duchess of Bourbon's Bed Chamber and how it is presented at the Louvre

\section{Frédéric Dassas}

\section{OpenEdition}

Journals

\section{Édition électronique}

URL : http://journals.openedition.org/insitu/24796

DOI : 10.4000/insitu.24796

ISSN : 1630-7305

\section{Éditeur}

Ministère de la Culture

\section{Référence électronique}

Frédéric Dassas, «Les témoignages du lit de la chambre de la duchesse de Bourbon au PalaisBourbon et leur présentation au Louvre », In Situ [En ligne], 40 | 2019, mis en ligne le 02 octobre 2019, consulté le 10 décembre 2020. URL : http://journals.openedition.org/insitu/24796 ; DOI : https:// doi.org/10.4000/insitu.24796

Ce document a été généré automatiquement le 10 décembre 2020.

\section{c) $(9)$}

In Situ Revues des patrimoines est mis à disposition selon les termes de la licence Creative Commons Attribution - Pas d'Utilisation Commerciale - Pas de Modification 4.0 International. 


\title{
Les témoignages du lit de la chambre de la duchesse de Bourbon au Palais-Bourbon et leur présentation au Louvre
}

\author{
The Bed from the Duchess of Bourbon's Bed Chamber and how it is presented at \\ the Louvre
}

Frédéric Dassas

Nous sommes très reconnaissant à Muriel Barbier et Nicolas Courtin de nous avoir donné l'occasion de présenter la communication qui est à l'origine de cet article. Nos remerciements s'adressent également à Jean Vittet et Thomas Bohl, qui ont rendu possible l'étude des cartons conservés au Mobilier national, ainsi qu'à Sébastien Ragueneau et Michel Chauveau, avec lesquels ont été conduites les recherches en vue de l'établissement du projet muséographique. La conduite de ce dernier a été rendue possible grâce à un mécénat exceptionnel de la Société des amis du Louvre. Notre gratitude s'adresse tout particulièrement à Jacques Garcia et à Sébastien Fumaroli, pour leur intérêt constant à l'égard de notre travail relatif à la grande chambre de l'hôtel de Chevreuse. Au sein du département des Objets d'art, nous remercions tout particulièrement Marie-Hélène de Ribou et Catherine Voiriot pour leur aide et leur collaboration précieuses.

Parmi tant de détails précieux que nous livre Luc Vincent Thiéry dans le second volume de son Guide des amateurs et des étrangers voyageurs à Paris publié en 1787, se trouve la mention de deux chambres de parade qu'il avait jugées suffisamment remarquables pour être dignes d'une attention particulière. Elles étaient situées à quelques centaines de mètres l'une de l'autre, la première à l'hôtel de Chevreuse (Luynes), rue SaintDominique, la seconde au Palais-Bourbon, à l'extrémité occidentale de la rue de l'Université. Les descriptions sont sommaires. La première concerne l'hôtel de Chevreuse : «La chambre à coucher de parade est après; elle a été décorée sur les dessins de M. Moreau ${ }^{1}$, architecte du roi ; la dorure y est magnifique, le meuble, ainsi que le lit et la tenture du fond d'alcôve, sont en satin brodé ${ }^{2}$.» Quelques pages plus loin, 
on lit à propos de celle du Palais-Bourbon: "On entre ensuite dans la chambre à coucher, tendue l'hiver de tapisseries des Gobelins, avec des médaillons coloriés, exécutés d'après Boucher, ainsi que le lit qui est d'un genre neuf ${ }^{3}$.» Les hasards de l'histoire font que les principaux témoignages de l'une et de l'autre se trouvent aujourd'hui réunis dans une même salle au Louvre, qui associe la dorure des boiseries de la première avec les tapisseries des Gobelins de la seconde (fig. 1). Ce sont ces tapisseries et leur histoire jusqu'à leur présentation actuelle, en particulier celles du grand lit à la duchesse «d'un genre neuf » qui en formait la partie la plus spectaculaire, qui nous retiendront ici.

\section{Figure 1}

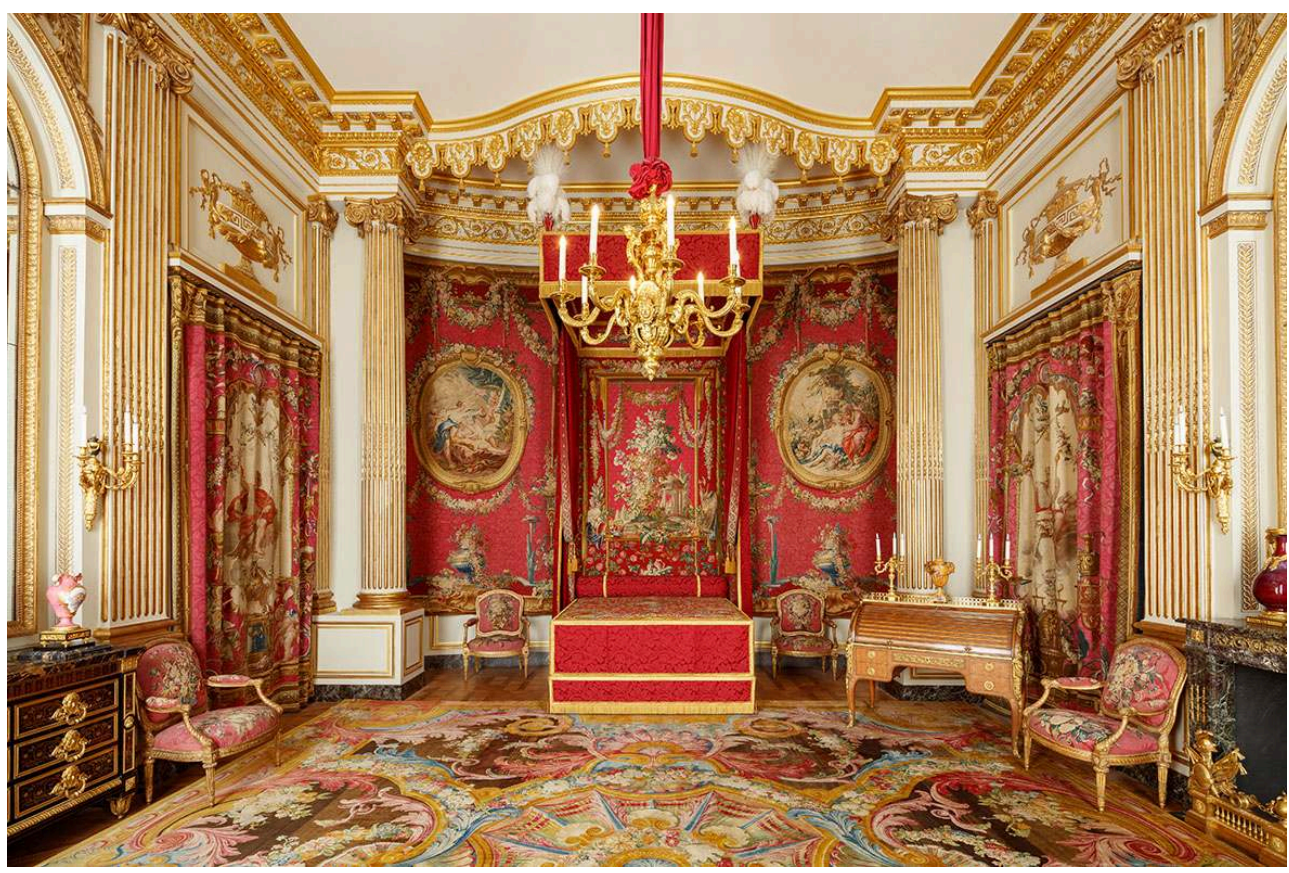

Vue de la salle 622 du département des Objets d'art au musée du Louvre, présentant les boiseries de l'ancienne chambre de parade de l'hôtel de Chevreuse (Luynes) et les éléments subsistants du décor de tapisseries des Gobelins de la chambre de la duchesse de Bourbon à l'hôtel de Lassay.

Phot. Éric Sander, 2018. @ Musée du Louvre (dist. RMN-GP).

\section{La chambre de la duchesse de Bourbon}

2 Le Palais-Bourbon était à la fin du XVIII ${ }^{\mathrm{e}}$ siècle la propriété de Louis-Joseph de Bourbon, prince de Condé (1736-1818) mais la chambre qui nous occupe n'était pas située dans le vaste palais encore connu sous ce nom, construit pour Madame la duchesse ${ }^{4}$, fille de Louis XIV et grand-mère du prince. Elle se trouvait dans l'hôtel voisin, autrefois occupé par le marquis de Lassay, hôtel que le prince de Condé entreprit de faire somptueusement décorer de neuf après en avoir fait l'acquisition en septembre 1768. L'hôtel de Lassay ne comprenait qu'un seul grand appartement, implanté en rez-dechaussée, dont la chambre ouvrait par deux fenêtres sur la Seine. La pièce était ornée du somptueux décor textile mentionné par Thiéry, qui associait aux tapisseries murales et au lit un mobilier complet assorti. Ce décor avait été conçu à la demande du prince de Condé à partir de 1769 mais ce dernier en avait laissé l'usage à sa belle-fille, Louise 
Marie Thérèse Bathilde, fille du duc d'Orléans, épouse depuis 1770 de son fils le jeune duc de Bourbon.

L'origine de la conception des tapisseries dont le prince avait fait le choix remonte aux initiatives prises par Jacques Germain Soufflot à la fin des années 1750 dans le but de donner une nouvelle impulsion aux productions de la manufacture des Gobelins. En 1758 avait émergé l'idée de confier à François Boucher l'exécution d'une série de compositions mythologiques destinées à prendre place dans un appareil ornemental figurant un cadre illusionniste suspendu sur fond de textile feint ${ }^{5}$. La composition des alentours avait été confiée à Maurice Jacques (1712-1784). Dès 1761, le projet d'une chambre complète, associant tapisseries murales, lit et mobilier assorti, avait connu un début d'exécution dans l'atelier de basse-lisse de Jacques Neilson, à l'intention de François Henri d'Harcourt, comte de Lillebonne et futur duc d'Harcourt (1726-1802). Jean Vittet a retracé le destin de ce projet malheureusement avorté, dont il subsiste la réplique d'une esquisse (fig. 2 ) et une série de cartons ${ }^{6}$. Il fallut cependant attendre la fin de la guerre de Sept Ans, en 1763, pour voir les projets de tissages se concrétiser, dans un premier temps à destination de clients étrangers. Les premières commandes de la nouvelle tenture, connue sous le nom de Tentures de Boucher, furent passées à Neilson entre 1763 et 1769 par des clients anglais : le comte de Coventry, William Weddell, Sir Henry Bridgeman et Sir Lawrence Dundas.

Figure 2

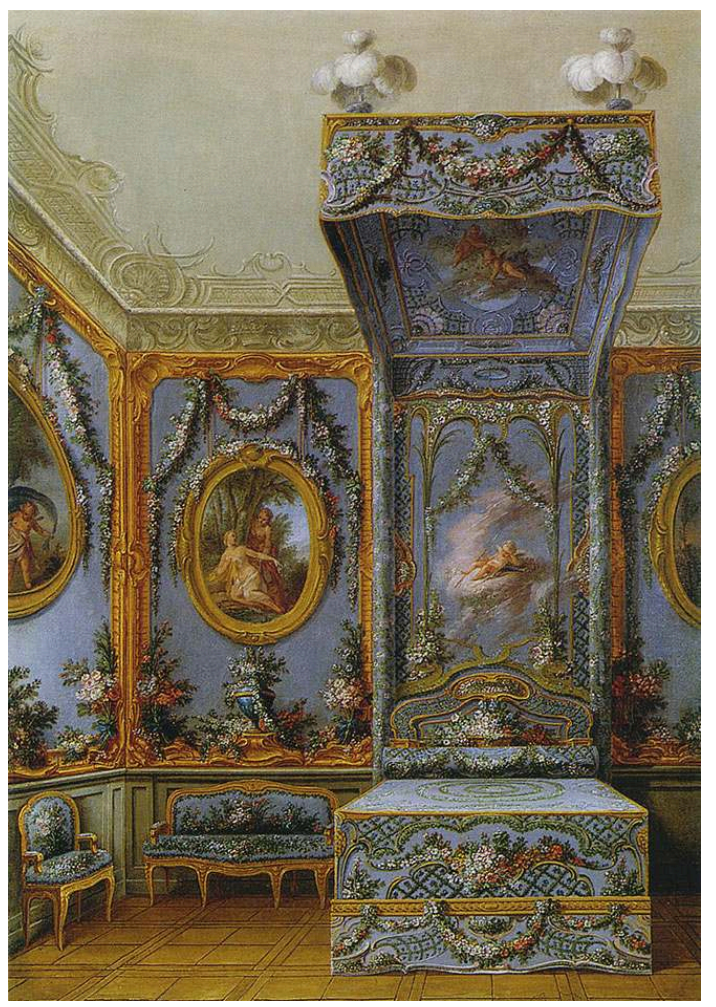

Maurice Jacques (d'après), projet pour le décor de tapisserie, (lit, tenture et couvertures de sièges), de la chambre du comte de Lillebonne, futur duc d'Harcourt, 1761. Localisation inconnue.

(c) Sotheby's 1996.

4 En envisageant la création d'une chambre de parade complète, le prince de Condé renouait avec les projets initiaux de Neilson. Ce dernier vit surtout dans la commande 
du prince la possibilité d'étendre à la France le succès remporté jusqu'alors à l'étranger et insista pour que le roi prenne à sa charge les frais de réalisation des nouveaux cartons $^{7}$. On comprend que par son ampleur, par le caractère prestigieux de son commanditaire et par le fait qu'il s'agissait du premier tissage destiné à un particulier français d'une tenture promise à un aussi large succès, la chambre de tapisserie de la duchesse de Bourbon, très admirée par ses contemporains, était destinée à marquer un jalon majeur de l'histoire du décor français.

5 L'élément le plus extraordinaire de cet ensemble était le lit à la duchesse qui occupait le centre de l'alcôve. Le document le plus aisé à interpréter pour s'en faire une idée est la description qu'en donne l'inventaire du mobilier du palais rédigé en $1779^{8}$, qu'il convient de citer largement pour prendre la mesure de la somptuosité exceptionnelle de ce meuble, véritable monument de sculpture. L'inventaire nous renseigne d'abord sur le détail de l'impériale :

Un lit à triple impériale dont celle du dessus est cartonnée et dorées en plain, les deux autres faites pour recevoir les étoffes, ornées de deux corniches très richement sculptées et dorées avec un couronnement à corniche ornée d'un ordre d'architecture avec dans chaque milieu un enfant, aux pieds du lit représentant les armes de S.A.S. et les deux autres représentant le chiffre, lesd. enfants accompagnés de six fortes guirlandes en bois richement sculpté, aux quatre coins de lad. impériale quatre socles recevant quatre panaches et aigrettes, aux deux coins du pied de lad. impériale deux culs de lampe recevant les glands et cordons.

Viennent ensuite les détails concernant la couchette :

Le bois de lit de sept pieds de long sur six pieds de large, le chantourné de sept pieds de haut orné d'une figure à grandeur nature, de branches de laurier et guirlandes de fleurs, le montant dudit chantourné en console et corniche avec une pomme de pin à chaque, au milieu dudit chantourné un médaillon dans lequel est le chiffre de Madame la duchesse. Les deux pieds en colonnes torses, et une pomme de pin à chacun.

7 Il est ensuite précisé : «Tous les bois de laditte impériale et de la couchette richement sculptés et dorés en différentes couleurs d'or » avant que ne soient plus rapidement décrites la garniture textile et la passementerie du meuble d'hiver : "L'étoffe dudit lit savoir l'impériale, pentes en dedans et en dehors, le dossier, quatre bonnes grâces, la courtepointe, les trois soubassements et le petit dossier chantourné en tapisserie des Gobelins fons damas cramoisy, orné de fleurs et guirlandes, le tout orné d'une très grande frange et bordée d'un mollet le tout en or faux surdoré. »

Le mémoire du sculpteur Charles Lachenait, très détaillé, précise ce qu'il en est de l'iconographie, en particulier de la figure de l'Immortalité portée sur des nuées qui surmonte le chantourné et du Génie de la guerre ornant de fleurs le médaillon du Grand Condé. Il ne laisse aucun doute sur la richesse extrême de l'appareil ornemental : les mentions de branches de laurier, de palmes, d'un casque, d'un sabre, et de trompettes entourées d'une couronne d'olivier confirment le caractère résolument guerrier du répertoire, tempéré de guirlandes de fleurs, de festons et de toutes sortes de rubans, de feuillages et de branchages : feuilles d'acanthes, feuilles d'eau, feuilles de persil, branches de roses, branches de jasmin, bouquets de fleurs, entremêlés de pommes de pin, de perles enfilées, de listels, d'oves, de linges, de graines et de glands. $\mathrm{Au}$ centre de chaque pan de la couchette se trouvait un mufle de lion coiffé d'une coquille, dont l'emplacement se retrouve dans le dessin des cartons. 
9 Les médaillons des quatre tapisseries qui ornaient l'alcôve étaient consacrés à des représentations de Vertumne et Pomone, l'Amour et Psyché, Aurore et Céphale et Vénus sur les eaux. Le mobilier de menuiserie associé comptait douze fauteuils, deux bergères, un écran et un paravent, tous couverts en tapisseries à bouquets de fleurs sur le même fond de damas cramoisi que les tapisseries. Ses bois avaient été exécutés par Louis Charles Carpentier et sculptés par Lachenait. La chambre abritait d'autres splendides meubles et objets. Le mobilier d'ébénisterie avait été exécuté par JeanFrançois Leleu (1729-1807) et comprenait une grande commode, aujourd'hui replaquée, conservée à Londres dans la collection Wallace ${ }^{10}$ et deux commodes d'entrefenêtres qui se trouvent au château de Versailles ${ }^{11}$. Une délicate garniture de vases de porcelaines de Sèvres à fond rose, achats antérieurs du prince, avait tout naturellement trouvé sa place dans ce décor aux harmonies colorées faites pour l'accueillir. Elle se composait d'un vase "vaisseau à mât ", de deux vases "à tête d'éléphant ", tous trois aujourd'hui conservés dans les collections du Metropolitan Museum of Art de New York ${ }^{12}$, et de deux vases « à oreilles » qui sont au Louvre ${ }^{13}$.

\section{Le devenir du décor de tapisseries}

10 C'est Christian Baulez qui a reconstitué les premières étapes de la vie de cet ensemble après le départ en émigration du prince de Condé14. Ce qui n'a pas été retenu de l'ameublement du palais par la Commission des arts est mis en vente sur place, à partir du 10 avril 1794. L'annonce de la vente mentionne la présence de «beaux meubles, lits, de tapisserie des Gobelins, meubles en tenture pareille... », puis le détail des vacations, à la date du 22 avril matin, apporte les précisions concernant « le lit en tapisserie des Gobelins, fond camayeu, quatre pièces de tenture de la même fabrique et du même fond, sujets mythologiques, garnitures de fauteuils et bergères, rideaux de lit et gros de Tours cramoisi ». Les tapisseries et les garnitures du mobilier sont mentionnées mais pas les bois ${ }^{15}$.

11 Un an plus tard, le 19 avril 1795 une annonce du Magasin de la Réunion des Arts propose à la vente « un lit à la polonaise composé d'un fond avec sa calotte, de deux grandes pentes, d'un dossier, d'une courtepointe, de 5 soubassements, un fronton, de 4 bonnes grâces, de 12 fauteuils complets, de deux bergères avec des coussins, de 6 feuilles de paravent, d'une feuille d'écran, le tout en tapisserie des Gobelins ${ }^{16}$ qui semble bien concerner notre ensemble. Il est clair qu'il ne s'agit encore une fois que des tapisseries, ce qui pourrait expliquer la confusion avec un lit à la polonaise puisque, nous le verrons, le panneau principal de la calotte était ovale. Quant aux tapisseries de l'alcôve, elles font rapidement retour au Garde-Meuble de la Couronne : en 1825 elles sont achetées à un certain Fauveau de La Tour pour la somme de 2500 francs. Leur provenance initiale est alors ignorée. Elles seront versées au Louvre par le Mobilier national en 1901.

12 La première étude des tapisseries de la chambre de l'hôtel de Lassay se trouve dans le IV ${ }^{\mathrm{e}}$ volume de l'État Général des tapisseries de la manufacture des Gobelins, publié par Maurice Fenaille en 1907. Les tapisseries du prince de Condé y figurent comme septième tissage des Tentures de François Boucher, le second tissage avec le second alentour, après celui que le roi avait offert à Joseph $\mathrm{II}^{17}$. Fenaille retrace l'histoire de deux des pièces depuis un reversement des Tuileries en 1863 et celle des deux autres jusqu'à un inventaire du Garde-Meuble de 1855. Le souvenir de l'achat de 1825, comme 
celui de la provenance Condé, s'est perdu. Fenaille évoque par ailleurs le décor du lit et la commande du prince de Condé, mais sans l'associer aux tapisseries. Il en fait remonter l'origine à la fin des années 1750, par association avec le projet de chambre de tapisserie du comte de Lillebonne et mentionne l'existence dans les réserves du Mobilier national d'un ensemble de cartons relatifs au projet ${ }^{18}$.

Qu'en était-il du reste du mobilier, et en particulier du lit, dont la trace était perdue depuis 1795 ? Ils réapparaissent quelques années plus tard, en Autriche, à Cumberland Schloss, la résidence qu'Ernest-Auguste de Brunswick, $3^{\text {e }}$ duc de Cumberland (1845-1923) s'était fait construire dans les années 1880 à Gmunden. Le père d'ErnestAuguste, Georges V, roi de Hanovre de 1851 à 1866, allié à l'Autriche lors de la guerre austro-prussienne de 1866, avait perdu son royaume à la suite de la victoire prussienne et trouvé refuge en Autriche auprès de l'empereur François-Joseph. En 1922, la galerie Duveen Brothers se rend acquéreur de l'ensemble des garnitures en tapisserie provenant de l'hôtel de Condé encore conservées dans la famille de Hanovre ${ }^{19}$. La correspondance échangée entre octobre 1951 et mars 1954, au moment de l'acquisition $\mathrm{du}$ fond de lit par le Louvre, entre Pierre Verlet, alors conservateur du département des Objets d'art, et Edward Fowles, propriétaire de la galerie Duveen depuis 1939, témoigne du fait que Duveen rattachait initialement un grand nombre de pièces à cet ensemble : un canapé, douze fauteuils, deux bergères, un paravent, un fond de lit, un bandeau ornemental et un ciel de lit, alors qu'il ne se trouvait pas de canapé au Palais-Bourbon. Fowles fait par ailleurs état d'une mention portée dans un inventaire attestant de la présence des tapisseries dans la famille de Hanovre depuis les années 1790.

Duveen, avant de les revendre, procéda à plusieurs interventions sur les tapisseries qu'il avait acquises. Les couvertures de six des fauteuils furent montées sur des bois estampillés par Gourdin complétés d'un canapé moderne. Ce premier ensemble, acheté par le banquier et collectionneur néerlandais Fritz Mannheimer (1890-1939), passa par la suite au Rijksmuseum ${ }^{20}$ où il est toujours conservé. Les couvertures des six autres fauteuils furent montées sur des sièges modernes de style rocaille. Ce second ensemble fut associé à quatre tapisseries des Tentures de Boucher provenant de la tenture donnée en 1782 par Louis XVI au Grand-Duc Paul ${ }^{21}$, que Duveen avait achetées au gouvernement soviétique, et passa à la Fondation Norton Simon, probablement au moment de la vente de l'ensemble du fonds de la galerie Duveen en 1964. Il fut rapidement revendu, accompagné de deux canapés, deux bergères, dix tabourets et un paravent six feuilles, associant de la même manière des bois modernes à des tapisseries anciennes dont la provenance initiale n'est pas attestée ${ }^{22}$.

Entre temps, le fond de lit avait été présenté en 1937 dans l'exposition du musée des Gobelins organisée par Guillaume Janneau et identifié comme le fond de lit du prince de Condé. Demeuré dans le fonds de la galerie Duveen, il est acheté par le Louvre en 1952. Il entre dans les collections assorti d'un bandeau ornemental, offert par Fowles comme provenant initialement du décor du lit de la duchesse de Bourbon ${ }^{23}$. À l'occasion de ces acquisitions, Pierre Verlet, dans un article dans la livraison de décembre 1953 de la Revue des arts, fait le point sur la question des tapisseries de la duchesse de Bourbon et livre un ensemble d'informations nouvelles ${ }^{24}$ : il propose d'identifier les quatre pièces des Tentures de Boucher conservées au Louvre avec celles de la chambre de parade de l'hôtel de Lassay; il envisage que le bandeau donné par Fowles soit un des soubassements du lit et mentionne l'existence de la courtepointe ainsi que des couvertures des sièges dans des collections américaines. À cette date, Pierre Verlet est 
en fait déjà sur la piste du ciel de lit. Fowles lui a signalé en mars 1952 l'existence d'un " canopy ", vendu par Duveen à Arnold Seligman et qu'il identifie dans une collection new-yorkaise. Pierre Verlet ayant réussi à convaincre sa propriétaire de le léguer au Louvre, ce dernier fragment entre dans les collections du musée en $1970^{25}$.

\section{Les cartons conservés au Mobilier national}

Le Mobilier national conserve une partie des cartons exécutés par Maurice Jacques pour le décor du lit, dont les mémoires subsistent par ailleurs ${ }^{26}$. Ces cartons portent des numéros à l'encre qui ne paraissent pas correspondre au mémoire de Jacques et sont de dimensions souvent sensiblement différentes de celles portées sur le mémoire, le peintre ayant parfois tendance à surestimer l'étendue de ses travaux. La plupart des cartons ne pose pas de problème d'identification : c'est le cas du fond de lit (GOB 245/1), de la pente extérieure de côté (GOB 245/2), des écharpes du baldaquin (GOB 245/3), des grands soubassements de côté et de face (GOB 245/8 et 245/9) et du traversin (GOB 245/10). Le cas des panneaux GOB 245/4 et 215/5 est plus délicat : ils peuvent être interprétés comme les modèles des pentes intérieures ou, plus probablement, des petits soubassements ${ }^{27}$. Les dimensions des cartons correspondant à la largeur du lit sont toutes inférieures à $200 \mathrm{~cm}: 181 \mathrm{~cm}$ pour le grand soubassement, $194 \mathrm{~cm}$ pour le grand dossier (fond) et 198 pour le traversin. L'écart est sensible avec les pièces correspondant à sa longueur : $236 \mathrm{~cm}$ pour la pente extérieure, $209 \mathrm{~cm}$ pour le grand soubassement de côté. Ces dimensions sont cohérentes avec celles du lit données par l'inventaire de 1779, qui sont de sept pieds sur six, soit environ 194,8 x 227,3 cm. Certains des modèles, malheureusement parmi les plus importants, sont manquants : celui de la courtepointe, celui du chantourné, ceux des calottes de l'impériale et ceux des enveloppes des rideaux et des bonnes grâces.

La confrontation entre les cartons du Mobilier national, le mémoire de Jacques, le mémoire de Lachenait et l'inventaire de 1779 apporte des éléments utiles à l'interprétation des tapisseries du Louvre.

Il est d'abord manifeste que le fond de lit a été agrandi puisque ses dimensions actuelles sont de $311 \mathrm{~cm}$ en hauteur sur 228 en largeur. Il a été augmenté sur chaque côté par des branchages de roses et complété dans sa partie inférieure pour masquer la surface originellement dissimulée par le chantourné, dont l'emprise est lisible sur le carton. On comprend bien l'intérêt de cette transformation qui vise à donner à un fragment de décor de lit l'apparence d'un panneau autonome. Il est possible que ces opérations aient été effectuées par rentrayage d'autres parties du décor. 


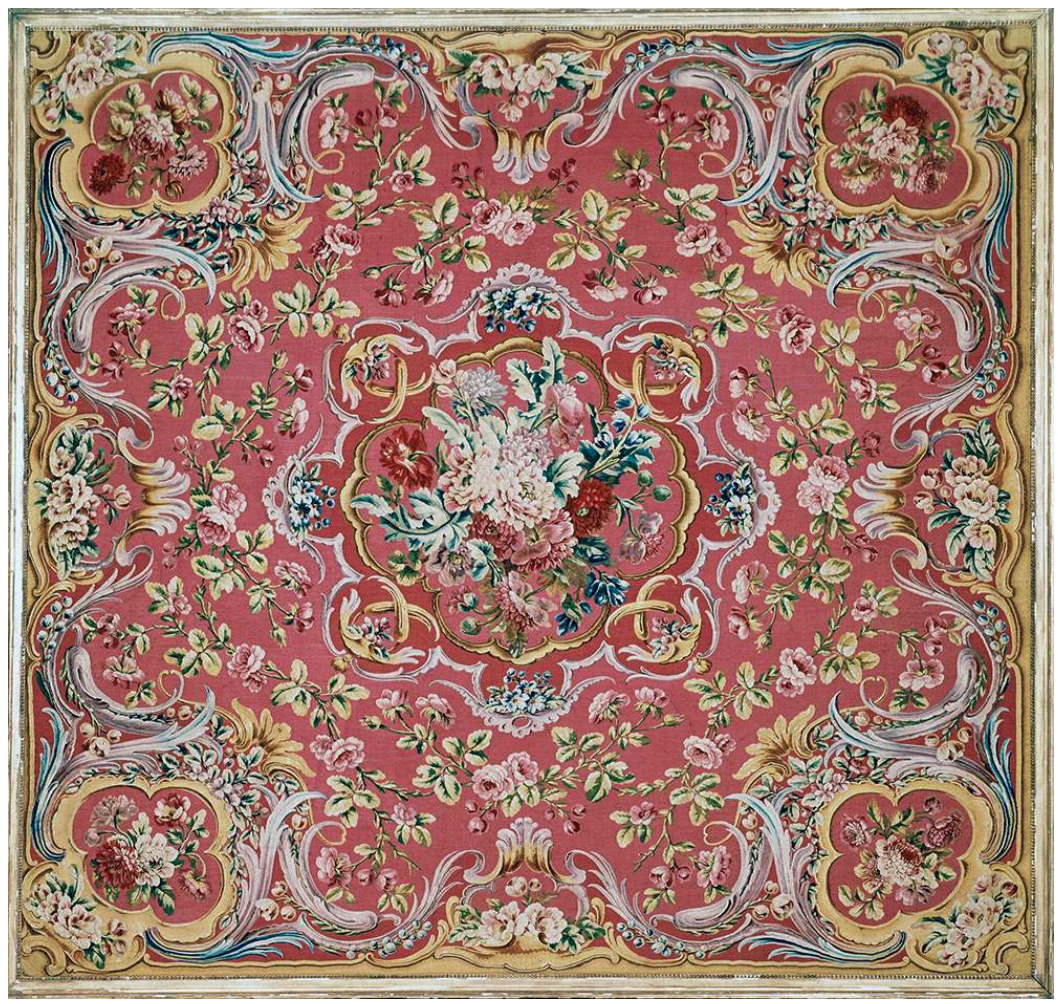

Panneau de tapisserie autrefois considéré comme provenant de l'impériale du lit de la duchesse de Bourbon à l'hôtel de Lassay. Probablement de la manufacture des Gobelins, vers 1760-1770. Paris, musée du Louvre, OA 10405.

Phot. (C) RMN-Grand Palais (musée du Louvre), 1970.

19 L'impériale pose des problèmes d'un autre ordre (fig. 3). Le mémoire de Lachenait ne laisse pas de doute sur le fait que l'intérieur de l'impériale n'était pas simplement rectangulaire mais organisé à partir de deux ovales superposés : «L'impériale du lit est d'une forme quarrée à l'extérieur et ovale dans l'intérieur. [...]. Toutes les moulures de cet ovale ont été prises dans la masse du bois. [...] Le second ovale fait un corps de plusieurs moulures qui forme un cordon. La principale partie de ce cordon est orné de bouquets de fleurs tournant autour d'une baguette ${ }^{28} »$. Les précisions de Lachenait sont à cet égard cohérentes avec la description du lit faite dans l'inventaire de 1779, qui mentionne un lit à triple impériale : manifestement une rectangulaire et deux ovales.

Si le panneau conservé au Louvre, qui est rectangulaire, ne peut être le ciel du lit, peutil en être la courtepointe? Il est intéressant de noter que la correspondance échangée entre E. Fowles et P. Verlet témoigne de positions successives et contradictoires sur cette question ${ }^{29}$. Le 31 octobre 1951, Fowles fait l'envoi au Louvre de la photographie du fond de lit, précisant que la seule pièce susceptible de provenir du lit dont il ait connaissance est un bandeau. Le 11 mars 1952, en réponse à Pierre Verlet qui s'était enquis de l'existence de rideaux, il révèle qu'il existerait également un « canopy » vendu par Arnold Seligman à une "Mme Strauss (sic)», qu'il n'identifie pas alors avec précision. C'est un an plus tard, après l'acquisition du fond de lit par le Louvre, que les courriers de Fowles font état d'un premier changement: dans celui du 2 novembre 1953, il fait à nouveau mention du «ciel de lit » vendu à Mme Strauss puis, dans celui $\mathrm{du} 23$, après qu'il a réussi à entrer en contact avec sa propriétaire (il s'agit de Mme Jesse Isidor Straus, née Irma Nathan (1877-1970), dont l'époux avait été ambassadeur en 
France entre 1933 et 1936), il n'est plus question d'un «ciel de lit» mais d'un « rose tapestry bed cover». Fowles précise qu'il est désormais encadré et accroché dans l'escalier de l'appartement de Mme Straus, 720 Park Avenue. C'est manifestement à ce panneau que fait référence la «courtepointe» conservée dans une collection américaine, dont Pierre Verlet fait mention dans son article de décembre 1953. Lorsque, le 27 janvier suivant, il fait l'envoi de cet article à Mme Straus, il prend soin d'ajouter : «Vous possédez, je crois, m'a-t-on dit, le couvre-lit de cet ensemble. » C'est également comme "bed cover » que Fowles fait référence au panneau Straus dans une lettre à Pierre Verlet du 24 mars 1954. Enfin, le 24 juillet 1970, dans un courrier adressé au directeur du Louvre André Parrot, suite à la délivrance du legs du panneau par Mme Straus, la position de Pierre Verlet n'a pas changé : il s'agit toujours de «la courte-pointe en Gobelins à fond cramoisi commandée en exemplaire unique par le prince de Condé en 1770 pour la chambre d'apparat du Palais-Bourbon. » Pour quelle raison tous les documents postérieurs ne feront-ils de nouveau référence qu'à une "impériale ", nous ne le savons pas. C'est sous cet intitulé que le panneau est inscrit dans l'inventaire du musée, sous le $n^{\circ} 10405$, et c'est en position de ciel de lit qu'il fut présenté dans les salles du département jusqu'en 2005 (fig. 4).

Figure 4

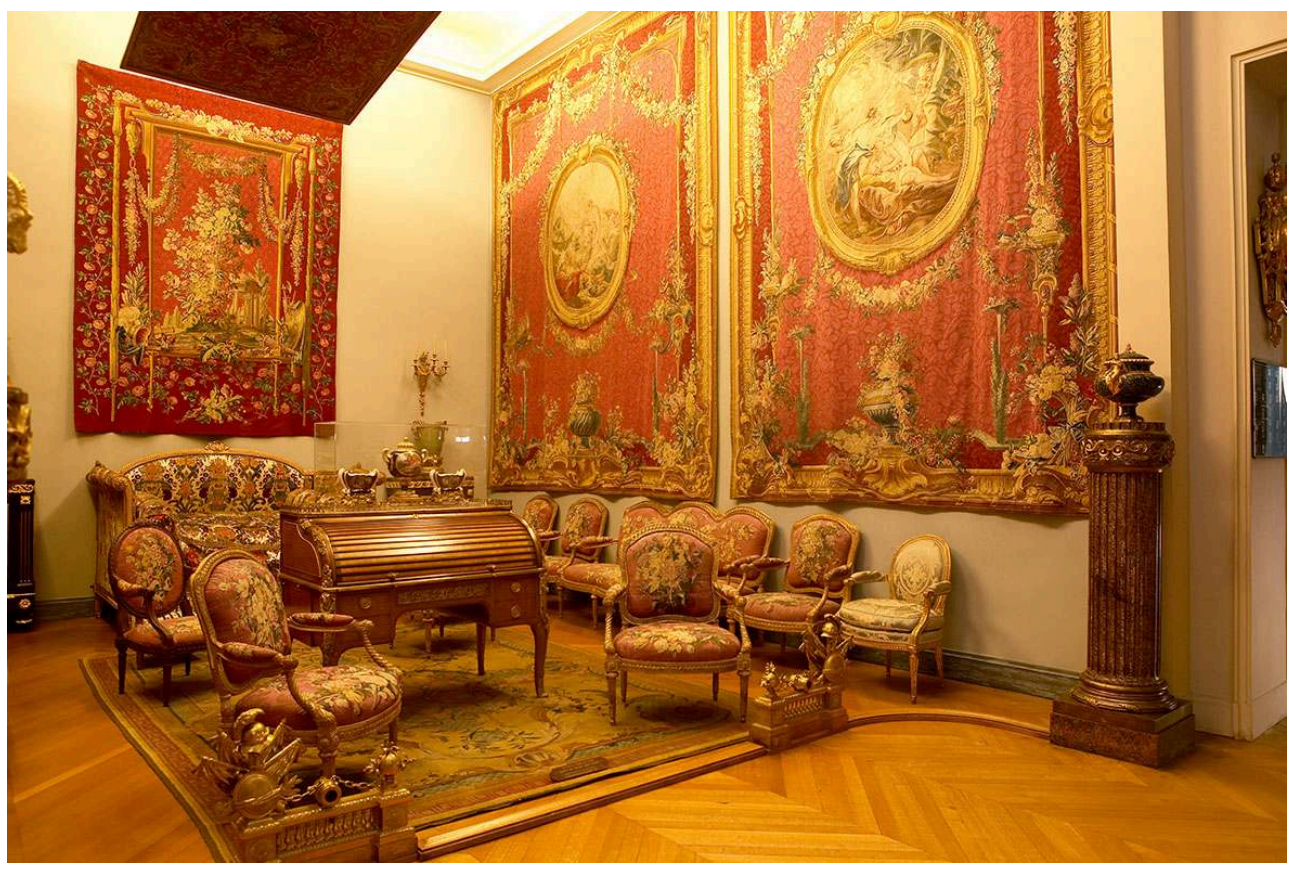

Les tapisseries, le fond de lit et le ciel de lit dans les anciennes salles du département des Objets d'art au Louvre.

Phot. Pierre Ballif, 2005. @ Musée du Louvre.

21 L'identification du panneau du Louvre avec la courtepointe du lit se heurte cependant à de sérieux obstacles. Le premier est la difficulté à l'accorder avec la description faite par Jacques dans son mémoire, qui fait état d'une riche bordure et de fleurs entrelacées et formant des festons que l'on ne retrouve pas sur notre panneau. Mais de manière plus générale, c'est la discordance stylistique entre ce panneau et les autres tapisseries, en particulier le fond de lit, qui est frappante : le style des motifs d'encadrement est sensiblement différent et la couleur de fond se distingue par une nuance plus pâle et 
plus rosée. Force est de reconnaitre que cette tapisserie ne provient probablement pas du décor de lit de la duchesse de Bourbon.

Reste enfin la question du bandeau ornemental offert par Fowles en 1952. S'il fait partie du lit, il ne pourrait s'agir que d'une pente intérieure. Il s'apparente davantage au fond de lit qu'au reste des cartons, par ses motifs de carquois, son harmonie colorée et le travail de ses fonds mais, là encore, il est difficile de l'accorder avec la description du mémoire de Jacques qui ne mentionne pas de carquois mais des guirlandes de fleurs attachées à des agrafes. Il faut garder à l'esprit que l'identification de ce bandeau ne remonte pas au-delà de la certitude qu'avait Edward Fowles qu'il était «venu avec le mobilier », mais cela trente ans après la transaction passée avec le duc de Cumberland. Nous avons vu que Fowles pensait de même de la garniture du canapé ainsi que du panneau de Mme Straus. On sait par ailleurs l'importance des manipulations effectuées sur les garnitures de sièges par la galerie Duveen avant leur revente. Il nous semble qu'à ce jour la provenance Condé de ce bandeau ne devrait être retenue qu'à titre d'hypothèse.

\section{La présentation des tapisseries au Louvre}

Nous avons exposé ailleurs la démarche d'ensemble qui avait été la nôtre dans la conduite de la restauration des boiseries de la chambre de parade de l'ancien hôtel de Chevreuse et les choix d'ameublement qui l'avaient accompagnée ${ }^{30}$. Dans le cas du lit, il était manifeste, au vu des éléments que nous venons d'exposer, que notre propos ne pouvait viser à la reconstitution d'un lit historique. Le terme de "lit muséographique ", que nous avons avancé, paraît assez justifié dans la mesure où les questionnements initiaux relevaient avant tout de problèmes de présentation de collection: comment concevoir la nouvelle installation des boiseries de la chambre, quel mobilier associer avec ces boiseries, dans quel cadre penser la réunion des meubles de tapisserie et les tapisseries murales relevant d'ensembles homogènes et, finalement, comment exposer les fragments associés au lit de la duchesse de Bourbon. La possibilité d'une présentation faisant référence à un lit à la duchesse émergea en grande partie en raison de facteurs qui lui étaient étrangers: en premier lieu la décision de remonter les boiseries de l'hôtel de Chevreuse en accord avec leur disposition originale de chambre de parade (fig. 5), en second lieu la volonté d'y réunir l'ensemble exceptionnel de tapisseries à fond cramoisi, constitué par les tapisseries Condé, le mobilier de Nicolas Heurtaut provenant du château de La Roche-Guyon et une paire de portières des Dieux à fond cramoisi assortie aux tapisseries d'alcôve. 


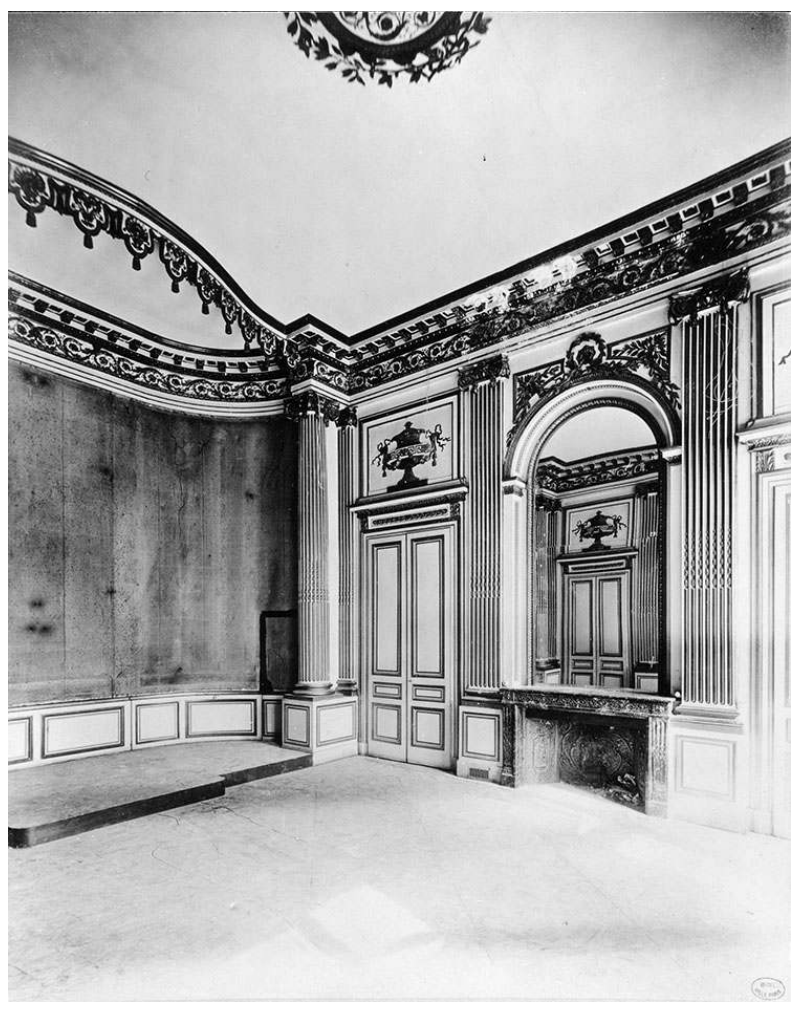

Vue de la chambre de parade de l'hôtel de Chevreuse (Luynes) avant le démontage des boiseries et la destruction de l'hôtel. Documentation du département des objets d'art.

(c) Musée du Louvre.

Si l'on se place du point de vue de la logique de la chambre du duc de Chevreuse, il ne fut jamais question de tenter une évocation de son somptueux lit de satin brodé dont on connaît l'aspect général grâce à une prisée faite en $1771^{31}$. Si l'on se place du point de vue de la cohérence des tapisseries, un retour au lit de l'hôtel de Lassay et à son extraordinaire décor sculpté était également impossible à envisager. Le document livrant les informations les plus utiles se révéla être finalement le projet de chambre du comte de Lillebonne de 1761, mentionné plus haut, avec son lit au dessin très sobre, reposant essentiellement sur la beauté des tapisseries. La conception du lit lui-même, fruit du travail conjoint de Sébastien Ragueneau, de Jacques Garcia et des équipes du Louvre, s'est d'abord présentée comme un entremêlement de problèmes de dimensions et de proportions, dont les trois principaux furent le défaut de profondeur de l'alcôve, à laquelle il manque une trentaine de centimètres, le rapport de hauteur entre la pièce et les tapisseries, initialement conçues pour prendre une place dans un volume plus vaste que ne l'était la chambre de l'hôtel de Chevreuse, enfin la largeur du grand dossier, agrandi de plusieurs dizaines de centimètres par rapport à ses dimensions d'origine.

Pour toutes ces raisons, ce lit présente un certain nombre de particularités remarquables: il est dépourvu de chantourné, il est dépourvu de bonnes grâces intérieures, sa couchette est sans doute trop basse, il rassemble des éléments de tapisseries d'origines différentes dont certains ont été modifiés, il mélange tapisseries de lice et soieries de façon très inhabituelle. Les dimensions de la couchette ont été déterminées par celles du panneau placé en courtepointe et sa hauteur établie pour s'accorder au positionnement des tapisseries murales. La hauteur du baldaquin 
correspond au déploiement du grand dossier, dont les côtés ont été repliés vers l'avant afin que soit intégrée leur largeur excessive. Les choix des étoffes de complément ont été faits afin que soit respectée la hiérarchie initiale entre tapisserie et damas, le damas se substituant aux premières, le gros de Tours au second et le taffetas au pékin des doublures. Le motif de fond du damas tissé spécialement par la maison Prelle a été repris des fonds damassés des tapisseries de l'alcôve (fig. 6).

\section{Figure 6}

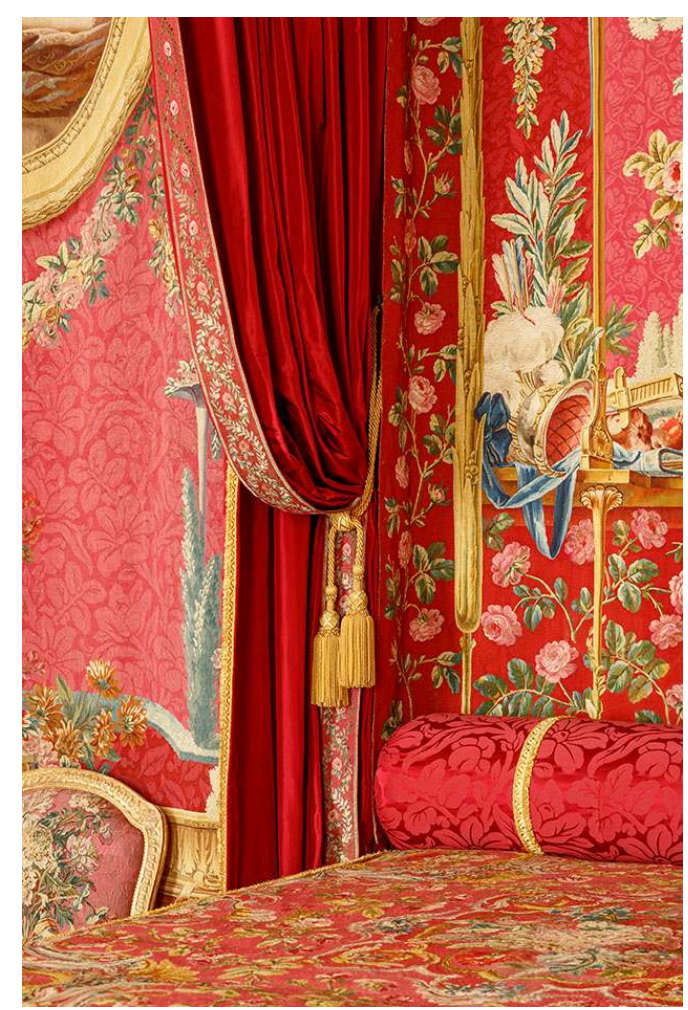

Détail de la présentation des différentes tapisseries associées au décor de la chambre de l'hôtel de Lassay.

Phot. Éric Sander, 2018. @ Musée du Louvre (dist. RMN-GP).

Comme nous souhaitions nous abstenir de toute intervention dans le domaine du bois sculpté, notre lit est d'une simplicité qui a peu à voir avec la richesse des grands lits d'apparat de son temps; il est même dépourvu de pieds normaux puisqu'il ne peut reposer sur ses pieds avant en raison de la présence d'un tapis de savonnerie. Il répond cependant aux missions que nous lui avons fixées: rendre justice aux témoignages exceptionnels de la chambre de l'hôtel de Lassay, en particulier le fond de lit, véritable chef-d'œuvre unique en son genre, contribuer à offrir une idée tangible de ce que furent les grandes pièces d'apparat à meuble de tapisserie et achever de donner tout leur sens aux boiseries de la chambre de parade de l'hôtel de Chevreuse. 


\section{NOTES}

1. - Pierre-Louis Moreau(-Desproux) (1727-1794), maître général des Bâtiments de la Ville de Paris.

2. - THIÉRY, Luc Vincent. Guide des amateurs et des étrangers voyageurs à Paris. Paris : Hardouin et Gattey, 1787, vol. 2, p. 528.

3. - Ibid., p. 601.

4. - Louise-Françoise de Bourbon, « Mademoiselle de Nantes ", duchesse de Bourbon (1673-1743).

5. - VITTET, Jean. Les Gobelins au siècle des Lumières. Un âge d'or de la manufacture royale. Cat. exp., Paris, galerie des Gobelins, 8 avril 2014-18 janvier 2015. Paris : Swan, 2014, p. 221.

6. - Ibid., p. 224.

7. - FENAILLE, Maurice. État général des tapisseries de la manufacture des Gobelins depuis son origine jusqu'à nos jours. T. IV, Dix-huitième siècle. Deuxième partie, 1737-1794. Paris : Imprimerie nationale, 1907, p. 401.

8. - Chantilly, Archives du musée Condé. Inventaire général des meubles et effets appartement au prince de Condé [...] (1779), AC 2 AC 027.

9. - Chantilly, Archives du musée Condé, AC 1 AC 006 (1772).

10. - F 246. Voir le site: https://wallacelive.wallacecollection.org/eMP/eMuseumPlus? service=direct/1/ResultListView/result.t1.collection_list.

$\$$ TspTitleLink.link\&sp=10\&sp=Scollection\&sp=SfieldValue\&sp=0\&sp=0\&sp=2\&sp=SdetailList\&sp=0\&sp=Sdetail\&sp=0\&sp=F\&sp=T\&sp=2 [ 11. - V 828 et 1149. Voir le site: http://collections.chateauversailles.fr/\#047c0b3dfc0a-4a6f-87d3-00fa62daabb9 [consulté le 17/07/2019].

12. - 58.75.89a, b (vase vaisseau à mât). Voir le site: https://www.metmuseum.org/art/ collection/search/202193; 58.75.90ab et 58.75. 91ab. (vases à tête d'éléphant). Voir les sites : https://www.metmuseum.org/art/collection/search/232706 et https://www.metmuseum.org/ art/collection/search/202194 [consultés le 17/07/2019].

13. - OA 11986 et 11987 . Voir le site: https://www.louvre.fr/oeuvre-notices/paire-de-vasesoreilles-en-premiere-grandeur-fond-rose?sous_dept=1 [consultés le 17/07/2019].

14. - Le Faubourg Saint-Germain. Palais-Bourbon, sa place. Paris : Institut néerlandais, 1987, p. 55.

15. - Annonces, affiches et avis divers, suppl. du 19 germinal an II [8 avril 1794], p. 7053 et 7054.

16. - Ibid., suppl. du 30 germinal an III [19 avril 1795], p. 3753.

17. - FENAILLE, Maurice. Op. cit., p. 278.

18. - Ibid., p. 399.

19. - Archives du département des Objets d'art, dossier d'œuvre OA 9846-9547.

20. - BAARSEN, Reinier. Paris 1650-1900. Decorative Arts in the Rijksmuseum. New Haven/Londres/ Amsterdam : Yale University Press/Rijksmuseum, 2013, p. 405.

21. - Futur empereur de Russie Paul I $\mathrm{er}^{\mathrm{r}}$ (1754-1801).

22. - New York, Parke-Bernett, 7 et 8 mai 1971 (lots $n^{\circ} 232$ et 233).

23. - OA 9546 (fond de lit) et OA 9547 (bandeau).

24. - La Revue des arts, $1953 \mathrm{n}^{\circ}$ 4, p. 243.

25. - OA 10405.

26. - Paris, AN, 01 2047. Nous sommes très reconnaissant à Jean Vittet de nous avoir très aimablement donné copie de ce document.

27. - VITTET, Jean. Op. cit., p. 349.

28. - Chantilly, Archives du musée Condé. Inventaire général des meubles et effets appartenant au prince de Condé [...] (1779), AC 2 AC 027.

29. - Archives du département des Objets d'art, dossier d'œuvre OA 9846-9547. 
30. - DASSAS, Frédéric. "Trois chambres pour un lit». L'Estampille/L'Objet d'art, $\mathrm{n}^{\circ} 538$, octobre 2017, p. 54.

31. - Paris, AN, MC/CXII/743, 24 octobre 1771.

\section{RÉSUMÉS}

Le musée du Louvre conserve un ensemble exceptionnel de tapisseries des Gobelins provenant du décor de la chambre de la duchesse de Bourbon au Palais-Bourbon: une suite de quatre tapisseries murales exécutées d'après des cartons de Maurice Jacques et François Boucher et trois panneaux traditionnellement associés au grand lit à la duchesse qui formait le principal ornement de la pièce. Ces tapisseries furent dispersées à la Révolution puis progressivement réintégrées au sein des collections nationales par des achats s'échelonnant entre 1825 et 1970. Les panneaux du lit ont fait l'objet d'une étude récente en vue de leur exposition dans les salles rénovées du département des Objets d'art. La confrontation avec les cartons de Maurice Jacques, conservés au Mobilier national, et avec les indications fournies par les mémoires et inventaires du XVIII ${ }^{e}$ siècle, qui donnent des descriptions assez précises du lit, a permis de reconsidérer leur provenance et de proposer une présentation, fondée sur l'évocation d'un lit de parade, au sein du décor exceptionnel constitué par les boiseries de la chambre de parade de l'hôtel de Chevreuse (Luynes) rue Saint-Dominique.

The Louvre today holds an exceptional ensemble of Gobelins tapestries which come from the bed chamber of the Duchess of Bourbon at the Palais-Bourbon. It comprises a series of four wall tapestries executed after the cartoons of Maurice Jacques and François Boucher and three panels traditionally associated with the grand canopy bed which was the principal piece of furniture in this chamber. These tapestries were dispersed at the time of the Revolution then gradually reintegrated into the national collections by purchases between 1825 and 1970 . The bed panels have been the object of a recent study in preparation for their exhibition in the Louvre's renovated rooms devoted to art objects. The comparison with the cartoons by Maurice Jacques, held by the Mobilier national, and with indications given by eighteenth-century texts and inventories give relatively precise descriptions of the bed. This allows for a museum presentation which evokes a bed of state within the exceptional decor made up of the wood panelling of the state bed chamber of the Hôtel de Chevreuse (Luynes) in the rue Saint-Dominique in Paris.

\section{INDEX}

Mots-clés : tapisserie, cartons de tapisserie, chambre de parade, lit à la duchesse, courtepointe, Palais-Bourbon, Hôtel de Chevreuse (Luynes), Maurice Jacques, Jacques Neilson, Charles Lachenait, Comte de Lillebonne, Galerie Duveen, tentures de François Boucher, Fritz Mannheimer, Pierre Verlet, Mobilier national, Louvre, département des objets d'art

Keywords : tapestry, tapestry cartoons, state bed chamber, Duchess-style bed, canopy bed, quilt, Palais-Bourbon, Hotel de Chevreuse (Luynes), Maurice Jacques, Jacques Neilson, Charles Lachenait, Comte de Lillebonne, Galerie Duveen, hangings by François Boucher, Fritz Mannheimer, Pierre Verlet, Mobilier national, Louvre, department of art objects 
AUTEUR

FRÉDÉRIC DASSAS

Conservateur en chef au département des Objets d'art, musée du Louvre

Frederic.Dassas@louvre.fr 